\title{
Pulsed Neutron Dose Monitoring - A New Approach
}

\author{
Alfred Klett, Member, IEEE, and Albrecht Leuschner
}

\begin{abstract}
Radiation protection at accelerator facilities is frequently dealing with pulsed radiation fields and with high particle energies. Therefore a new method for pulsed highenergy neutron dose monitoring with an active device was developed and investigated. The concept is based on the idea of storing pulsed dose information in an instable nuclear state with a short half-life. High-energy neutrons are hitting on a ${ }^{12} \mathrm{C}$ target and are generating ${ }^{9} \mathrm{Li}$ nuclei with a half-life of $178 \mathrm{~ms}$. The daughter nuclide disintegrates with neutron emission. Berthold LB 6411 standard rem-counters were used as neutron detectors and as carbon targets. The data were recorded in time-resolved readout utilizing a newly developed data-logger, which auto-synchronizes with pulsed radiation fields. Measurements with pulsed high energy neutron fields were performed and calibrated and the analysis of the delayed neutrons provides neutron dose information without being affected by dead-time.
\end{abstract}

\section{INTRODUCTION}

$\mathrm{R}$ ADIATION protection at accelerator facilities requires area dose monitoring of gamma and neutron fields. Behind the shieldings of high-energy accelerators the main dose contribution is in many cases caused by high-energy neutrons with energies exceeding $20 \mathrm{MeV}$. Radiation fields at accelerators might not only have high energies, but sometimes they have also a characteristic time structure and are pulsed.

For routine neutron dose rate monitoring there are many instruments available on the market. But they cover usually only the energy range of classical nuclear physics between thermal energies and $20 \mathrm{MeV}$. At higher neutron energies conventional rem-counters are known to underestimate neutron doses considerably. Therefore for neutron dose monitoring in accelerator environments detection techniques with extended measuring range to higher neutron energies are required.

The measurement of pulsed radiation fields could suffer from dead-time effects of active radiation detectors. For instance proportional counters or scintillation counters could be affected by dead-time counting losses. As most remcounters utilize ${ }^{3} \mathrm{He}$ - or $\mathrm{BF}_{3}$-proportional counter tubes for thermal neutron detection, they have limitations in measuring

\footnotetext{
Manuscript received November 26, 2006

Alfred Klett is with BERTHOLD TECHNOLOGIES GmbH \& Co. KG, Calmbacher Strasse 22, 75323 Bad Wildbad, Germany

(e-mail: alfred.klett@Berthold.com).

Albrecht Leuschner is with DESY Deutsches Elektronen-Synchrotron, Notkestrasse 85, 22607 Hamburg, Germany

(e-mail: Albrecht.Leuschner@desy.de).
}

pulsed neutron fields. For moderator based instruments the moderation process is counteracting against dead-time effects. The delays of neutron moderation are typically ranging up to $100 \mu \mathrm{s}$. As the detected neutron events are spread out in time, dead-time effects are partly compensated for. But moderation won't be able to solve the problem for very short and intense radiation pulses.

Berthold Technologies a German manufacturer of radiation protection instrumentation and Deutsches ElektronenSynchrotron DESY in Hamburg have established a cooperation to address these two problems with high energies and pulsed neutron radiation fields in neutron area dose monitoring. The objective of this joint effort is the design of a completely new system, which should be appropriate for routine operation in accelerator laboratories and would become commercially available.

The new approach is resting upon an idea of DESY, which was already applied for patent [1], [2]. The concept is based on the idea of storing pulsed dose information in an excited nuclear state with a short half-life. Decay products of this intermediate short lived state are being detected in time resolved readout. These events are not subject to dead-time and the threshold neutron energy of the reaction ensures measurement of high-energy neutrons only.

\section{PRINCIPLES OF DETECTION AND DESCRIPTION OF THE INSTRUMENT}

For the implementation of this idea a special nuclear reaction was selected, where incident neutrons would generate on a target material sufficient amounts of a radioactive nuclide with a short half-live. The nuclear reaction ${ }^{12} \mathrm{C}(\mathrm{n}, \mathrm{X}){ }^{9} \mathrm{Li}$ was chosen. It has a reaction threshold of $40 \mathrm{MeV}$ and a relatively high cross-section of 0.2 mbarn at $100 \mathrm{MeV}$. The ${ }^{9} \mathrm{Li}$ nuclei which are produced by high energy neutrons in the target material carbon are not stable. The decay ${ }^{9} \mathrm{Li} \rightarrow{ }^{9} \mathrm{Be}^{*}+\beta^{-}+v$ has a half life of $178 \mathrm{~ms}$. The ${ }^{9} \mathrm{Be}^{*}$ nuclide instantaneously disintegrates ${ }^{9} \mathrm{Be}^{*} \rightarrow \alpha+\alpha+\mathrm{n}$ by emitting a fast neutron. Thus, these delayed neutrons are having a time spectrum relative to the prompt neutrons from the beam pulse, which corresponds to the decay pattern with $178 \mathrm{~ms}$ half life.

For the selected nuclear reaction an assembly is required with the target material carbon and with a neutron detector. We utilized for this purpose the Berthold LB 6411 neutron dose rate monitor, a widespread standard instrument. It is a conventional rem-counter for neutron energies below $20 \mathrm{MeV}$ 
with a total weight of $10 \mathrm{~kg}$. It has a moderating sphere with a diameter of $250 \mathrm{~mm}$ providing sufficient amounts of carbon as a target material for the generation of ${ }^{9} \mathrm{Li}$ nuclei. The LB 6411 detects thermal neutrons with a cylindrical ${ }^{3} \mathrm{He}$ proportional counter tube which is centered in the moderator sphere. In normal operation mode the LB 6411 is measuring ambient dose equivalent $\mathrm{H}^{*}(10)$ for neutrons from thermal energies up to $20 \mathrm{MeV}$. The response for bare ${ }^{252} \mathrm{Cf}$ is 2.83 counts $/ \mathrm{nSv}$. The energy dependent response was tuned with internal absorbing layers and it is $\pm 30 \%$ between $50 \mathrm{keV}$ and $10 \mathrm{MeV}$ neutron energy. A detailed description of this instrument together with MCNP results and calibration data measured at the Physikalisch-Technische Bundesanstalt (PTB) in Braunschweig was published earlier [3], [4].

In addition and parallel to this standard LB 6411 neutron dose monitor we used also another special version neutron probe $\mathrm{LB}$ 6411- $\mathrm{Pb}$ [5]. This version was recently developed for high energy applications for neutron energies up to $1 \mathrm{GeV}$. It has an additional external spherical layer of lead with a thickness of $10 \mathrm{~mm}$ to utilize spallation neutrons to enhance the response at high energies. The overall weight of this instrument is $35 \mathrm{~kg}$. It was calibrated in the CERF field at CERN in Geneva in Switzerland [6].

The time-resolved readout of the detectors was performed by a newly developed small data-logger Berthold LB 5360 . This data acquisition system is based on a Motorola 68340 CPU (max. $24.117 \mathrm{MHz}$ ). It has $1 \mathrm{MByte}$ flash memory, 128 kByte RAM, a real time clock and a serial RS232 port on board. There are two independent counting channels for rates up to $7.48 \mathrm{MHz}$. The experimental readout of the detectors was controlled by the real time clock in accurate time slices of $1 \mathrm{~ms}$. The system is auto-synchronizing with pulsed radiation fields by using a special threshold condition and accumulates the spectral distribution of the events in time. It auto cycles and transmits data to an external host computer or network through the serial RS232 port.

\section{IRRADIATION WITH High ENERGY NEUTRONS}

The instruments were irradiated with pulsed neutron fields at DESY. The fields were generated by a $7.5 \mathrm{GeV}$ proton beam in the transfer tunnel from the DESY III synchrotron to the PETRA storage ring. By switching of a bending magnet in the injection line a complete beam loss was generated in the shielding. It consisted out of the concrete walls of the tunnel and the hall and the soil in between and it had about $2.5 \mathrm{~m}$ of total thickness. Behind the shielding there was the irradiation platform inside the PETRA experimental hall. The proton beam had a repetition rate of $0.25 \mathrm{~Hz}$ with a microstructure of 10 bunches spaced at $96 \mathrm{~ns}$ length. The total width of one pulse train was almost $1 \mu \mathrm{s}$ and it had a total number of $10^{12}$ protons. On the irradiation platform the neutron energy spectrum is expected [7], [8] to be in equilibrium for normal concrete as shown in Fig. 1.

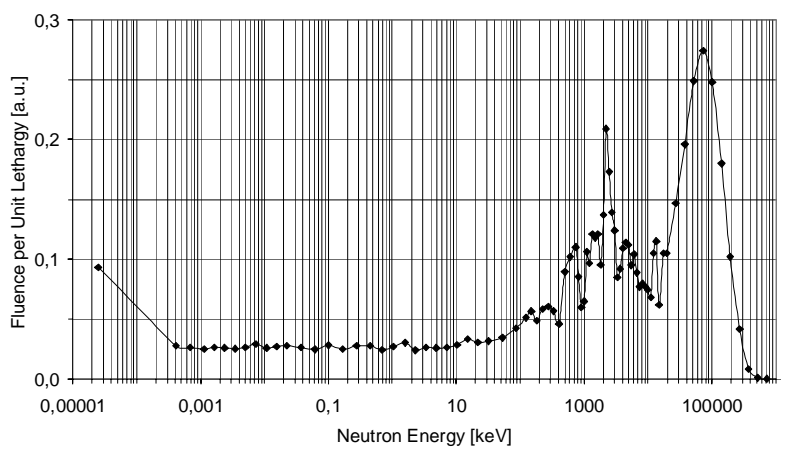

Fig. 1. Energy spectrum of the neutron fluence in lethargy representation

The experiment was performed on March 9, 2006. The detectors were irradiated for a period of 400 seconds with 101 consecutive bunch trains. The doses were monitored with several independent passive dose meters, which are commonly used at DESY. The TLD600/700 in a polyethylene cylinder and a Thorium radiator on Macrofol foil were calibrated to the whole neutron spectrum including high energies. A single bunch train generated a neutron dose of $50 \mu \mathrm{Sv}$ at the neutron probe's position in the experimental hall. The bubble detectors BD-PND produced by Bubble Technology Industries have a sensitivity of $13 \mu \mathrm{Sv} / \mathrm{bubble}$ measuring only the low energy part of the neutron spectrum below $20 \mathrm{MeV}$. The bubbles indicated per bunch train a dose of $27 \mu \mathrm{Sv}$. From these measurements it is concluded, that there was a contribution of $27 \mu \mathrm{Sv}$ from low energy neutrons and of $23 \mu \mathrm{Sv}$ from high energy neutrons adding up to a total dose of $50 \mu \mathrm{Sv}$ per bunch train for the whole neutron spectrum. The gamma dose was indicated by the TLD700 to be $7 \mu \mathrm{Sv}$ per bunch train.

\section{DATA ANALYSIS}

The result of the measurement are the accumulated delay time histograms of the LB 6411 and the LB 6411-Pb. The background was estimated at large delays of a few seconds and was subtracted in the spectra. The time spectra were then rebound in logarithmically equidistant intervals in a lethargy type of representation.

A simple fit function (1) with a fast and a slow component as a function of delay time $t$ was used to model the data. The fast component of this function with a fit parameter $\tau_{1}$ of $1.5 \mathrm{~ms}$ is describing those neutrons directly arriving from the source region. A thermalized neutron would for example travel across a distance of about $3 \mathrm{~m}$ within $1.5 \mathrm{~ms}$. The slow component of this fit function with a fit parameter $\tau_{2}$ of $245 \mathrm{~ms}$ is describing the delayed neutrons from the decay of the intermediate ${ }^{9} \mathrm{Li}$ nuclei.

$y=t \cdot\left[C_{1} \cdot \exp \left(-t / \tau_{1}\right)+C_{2} \cdot \exp \left(-t / \tau_{2}\right)\right]$ 




Fig. 2. Delay time distribution LB 6411 in lethargy representation

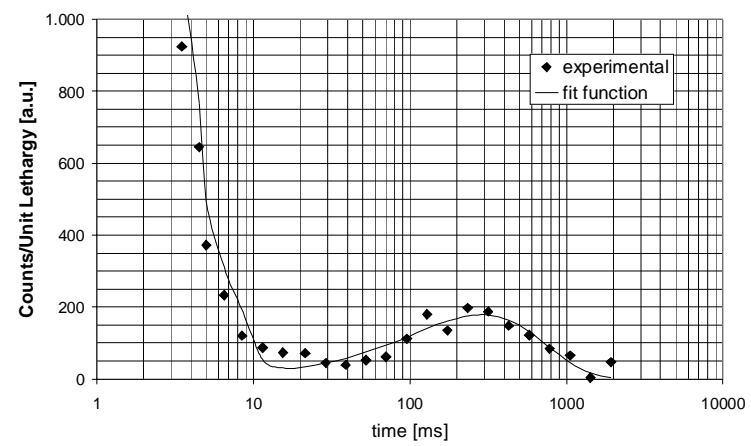

Fig. 3. Delay time distribution LB 6411-Pb in lethargy representation

These distributions together with the fit functions are shown in Fig. 2 and Fig. 3 for the LB 6411 and for the LB 6411-Pb respectively. On the left there are the fast tails of the prompt neutrons. The peaks with the ${ }^{9} \mathrm{Li}$ events are in this lethargy representation very nicely visible at the characteristic delay times. Per single shot there were with the LB 64116.0 counts detected and 4.8 counts with the LB $6411-\mathrm{Pb}$ resulting in a dose response of 0.26 counts $/ \mu \mathrm{Sv}$ respectively 0.21 counts $/ \mu \mathrm{Sv}$. The conventional response of the two remcounters was also determined. There was a very severe under response observed. All results are in Table I.

TABLE I

NEUTRON DOSE RESPONSE

\begin{tabular}{|l|r|r|l|}
\hline Neutron probe & LB 6411 & LB6411Pb & Units \\
\hline Time resolved analysis & & & \\
Neutron dose (high energies) & 23 & 23 & $\mu \mathrm{Sv}$ \\
${ }^{9}$ Li neutrons detected & 6.0 & 4.8 & counts \\
Neutron dose response & 0.26 & 0.21 & counts $\mu \mathrm{Sv}$ \\
\hline Conventional analysis & & & \\
Neutron dose (all energies) & 50 & 50 & $\mu \mathrm{Sv}$ \\
Total counts $(\Delta \mathrm{t}=3$ s) & 250 & 155 & counts \\
Conventional dose indication & 0.09 & 0.05 & $\mu \mathrm{Sv}$ \\
Relative dose response & $0.18 \%$ & $0.11 \%$ & \\
Factor of under response & 568 & 909 & \\
\hline
\end{tabular}

\section{CONCLUSIONS}

A new approach with time-resolved readout for the measurement of pulsed high-energy neutron doses was successfully tested and proven to work. The apparatus was irradiated at DESY. Pulsed dose information was stored by activation of the radioactive nuclide ${ }^{9} \mathrm{Li}$. Decay neutrons emitted from ${ }^{9} \mathrm{Li}$ can easily be identified and analyzed in the recorded delay time spectra. The calibration factor was determined to be 0.26 counts $/ \mu \mathrm{Sv}$. This sensitivity is sufficient for routine neutron dose rate monitoring in pulsed neutron fields. The method could be applied in accelerator environments with pulsed neutron radiation with repetition rates up to several $\mathrm{Hz}$.

These investigations also revealed that all common types of conventional rem-counters would fail in pulsed radiation fields with very sharp time structure and high intensity. We measured in normal operation mode severe dead-time effects. The dose was tremendously underestimated by up to three orders of magnitude.

\section{ACKNOWLEDGMENT}

The authors would like to thank M. Salmani, W. Clement and B. Mukherjee for the passive dosimetry and the DESY machine crew for the delivery of the proton beam.

\section{REFERENCES}

[1] DESY, "Method And System For Determining The Flow Of High-Energy Neutrons", WO 2006/097338 A1, PCT Patent Application, March 20, 2006, published Sep. 21, 2006

[2] DESY, "Verfahren und System zur Bestimmung der Fluenz hochenergetischer Neutronen", DE 102005012630 A1, German Patent Application, March 18, 2005, published October 5, 2006

[3] Klett Alfred and Burgkhardt Bertram, "The New Remcounter LB6411: Measurement of Neutron Ambient Dose Equivalent $\mathrm{H}^{*}(10)$ according to ICRP60 with High Sensitivity", IEEE Trans .Nucl. Sci., vol. 44, no.3, pp. 757-759, June 1997

[4] B. Burgkhardt, G. Fieg, A. Klett, A. Plewnia, B.R.L. Siebert, "The Neutron Fluence and $\mathrm{H}^{*}(10)$ Response of the New LB6411 Rem Counter", Neutron Dosimetry, Proc. 8th Symposium, Paris, Nov. 13-17 1995, Rad. Prot. \& Dos., vol. 70, no. 1-4, pp. 361-364, 1997

[5] Klett Alfred, Mayer Sabine, Theis Christian, Vincke Helmut, "A Neutron Dose Rate Monitor For High Energies", RadSynch'04, 3rd Int. Workshop On Radiation Safety of Synchrotron Radiation Sources, Proceedings, SPring-8, Hyogo, Japan, 17-19 Nov 2004

[6] Mitaroff A. and Silari M., "The CERN-EU High Energy Reference Field (CERF) Facility for Dosimetry At Commercial Flight Altitudes And in Space", Rad. Prot. \& Dos., vol. 102, no. 1, pp. 7-22, 2002

[7] Dinter H., Tesch K., Dworak D., "Studies On The Neutron Field Behind Shielding Of Proton Accelerators. Part I: Concrete Shielding". Nuclear Instruments \& Methods, A 368, 265-272, 1996

[8] Dinter H., Leuschner A., Tesch K., Dworak D., Loskiewicz J., "Calculation Of Hadron Yields Around Thick Targets And doses Behind Concrete Shielding Of High Energy Accelerators". Nuclear Instruments \& Methods, A 455, 460-469, 2000 